\title{
RECENT FLIGHT RESULTS OF THE TRMM KALMAN FILTER
}

\author{
Stephen F. Andrews \\ Aerospace Engineer \\ Flight Dynamics Analysis Branch \\ Guidance, Navigation, and Control Center \\ NASA Goddard Space Flight Center \\ Greenbelt, MD 2077I USA \\ Stephen Bilanow \\ Navigation Analyst \\ TRMM Science and Data Information System \\ Science Applications International Corp. \\ Greenbelt, $M D, 20771$
}

\begin{abstract}
The Tropical Rainfall Measuring Mission (TRMM) spacecraft is a nadir pointing spacecraft that nominally controls the roll and pitch attitude based on the Earth Sensor Assembly (ESA) output. TRMM's nominal orbit altitude was $350 \mathrm{~km}$, until raised to $402 \mathrm{~km}$ to prolong mission life. During the boost, the ESA experienced a decreasing signal to noise ratio, until sun interference at $383 \mathrm{~km}$ altitude made the ESA data unrcliable for attitude determination. At that point, the backup attitude determination algorithm, an extended Kalman filter, was enabled. After the boost finished, TRMM reacquired its nadir-pointing attitude, and continued its mission. This paper will briefly discuss the boost and the decision to turn on the backup attitude determination algorithm. A description of the extended Kalman filter algorithm will be given. In addition, flight results from analyzing attitude data and the results of software changes made onboard TRMM will be discussed. Some lessons learned are presented.
\end{abstract}

\section{Introduction}

The Tropical Rainfall Measuring Mission (TRMM) spacecraft is a joint NASANASDA mission that was launched on November 27, 1997 from Tanegashima Space Center, Japan. The spacecraft is three-axis stabilized, initially in a near circular $350 \mathrm{~km}$ orbit at $35^{\circ}$ inclination. The sensor complement includes a static Earth Sensor Assembly (ESA), two two-axis Digital Sun Sensors (DSS), a redundant Inertial Rate Unit (IRU), eight Coarse Sun Sensors (CSS), and two Three-Axis Magnetometers (TAM). The spacecraft is controlled with four Reaction Wheels Assemblies (RWA).lwelve thrusters (Reaction Engine Modules, REM), and three Magnetic Torquer Bars (MTB) for momentum unloading. In Mission Mode, which is the nadir pointing science configuration, attitude determination was nominally done with the ESA for roll and pitch, and integrated IRU rate for yaw.'

\section{Boost Description}

The purpose of raising TRMM's orbit from $350 \mathrm{~km}$ to $-400 \mathrm{~km}$ is to extend its mission life by reducing the fuel expenditure used to compensate for atmospheric drag. Extending the mission would provide the opportunity to possibly capture the transition to El Niño from neutral conditions that was missed in the Nov. 1997 launch. This would provide a less biased tropical rainfall climatology under a range of climate conditions. In addition, more observations are needed to confirm pollution/precipitation links observed by TRMM. Also, an extended mission enables integration of TRMM rainfall data into NASA global model analysis along with data from Terra, Aqua, and the ADEOS II. The approval was given for the boost in the summer of 2001, and the boost began on August 7 , 2001. The planning and execution of this activity is beyond the scope of this paper.

At the higher altitude, it was recognized that the performance of the ESA would be marginal, duc to the lower signal to noise ratio in the ESA detector heads. Contingency plans were developed to enable the Kalman filter during the boost activities if needed. On August 13,2001 , on a day between maneuvers, TRMM entered Safehold after a series of Failure Detection and Correction (FDC) events, triggered by DSS measurement faults and two ESA heads being marked bad. ${ }^{2}$ These FDCs triggered following three days of successful Delta-V Boost burns which placed the TRMM spacecraft into a new higher orbit of $386.90 \mathrm{~km}$ x $379.84 \mathrm{~km}$. At this new altitude, the Barnes static Earth Sensor Assembly (ESA) has a lower signal to noise ratio than at the operational $354 \times 347 \mathrm{~km}$ orbit. The ESA always produces spikes in roll and pitch position error during transitions between three and four quadrant control due to an anomalous behavior with the internal Offset Radiation Source (ORS). These transitions occur whenever there is predicted sun and/or moon quadrant interference. However, these spikes became much larger due to the increased sensitivity at the new altitude and on August 13, 2001, a larger than usual roll error was produced which eventually translated into yaw. As a result, the observatory was no longer pointing directly along the velocity vector, and there was an accumulated error that exceeded the FDC limit of 
$2^{\circ}$. Yaw gyro drift corrections commonly known as yaw updates normally occur twice an orbit centered around orbit noon using the Fore and Aft DSSs to correct for the yaw gyro drift. However, due to the large yaw error that was induced from the last ESA spike, the yaw update scheduled for 02:21:57 GMT did not occur and FDC Test 23 triggered, assuming there was a 'problem' with the DSS-A. Without a correction in yaw, this error continued to propagate through the orbit and as a result, the next yaw update did not occur either, sending the spacecraft to Sun Acquisition mode. The fact that two ESA quadrants were flagged bad also indicates that there was a large accumulated yaw error and the spacecraft was no longer properly pointing at the earth. After performing simulations for Sun Acquisition exit, three-minute Delta $\mathrm{V}$ burns, and a Yaw maneuver test while using the Kalman filter for attitude determination, the decision was made to recover back to normal mode and continue the boost activities without using the ESA. The extended Kalman filter algorithm was enabled on August 16, 2001.

\section{Algorithm Heritage}

The extended Kalman filter used on TRMM provides updates to the IRU propagated attitude and the IRU drift rate biases with TAM and DSS measurements. Other satellites have used a DSS and a TAM to calculate a deterministic attitude solution employing a TRIAD algorithm. ${ }^{3}$ For estimation attitude determination, typical spacecraft applications of a Kalman filter use a Star Tracker (ST), sometimes in concert with a DSS, to update the IRU propagated attitude. The Solar Maximum Mission (SMM), the Extreme Ultra Violet Explorer (EUVE), and the Rossi X-ray Timing Explorer (RXTE) use a Kalman filter with a DSS and an ST, for cxample.

Hashmall, Rokni, Sedlak, et. al, first demonstrated the feasibility of determining spacecraft attitude using only magnetometer and gyro data. ${ }^{4}$ Their analysis, based on flight data from the Upper Atmosphere Research Satellite (UARS) and the Extreme Ultra Violet Explorer (EUVE), showed that magnetometers, with gyros, could be used to determine attitude to an accuracy of better than $0.1^{\circ}, 1 \sigma$. This study, coupled with flight heritage, gave GSFC the confidence to design a contingency attitude determination mode utilizing the existing gyros, TAM and DSSs as backup attitude sensors.

The TRMM Kalman filter was adapted from RXTE Kalman filter, which was based on the Kalman filter developed for the Multimission Modular Spacecraft (MMS) by Murrel1 ${ }^{5}$, and described in Lefferts, Markley, and Shuster. ${ }^{6}$ The major changes to the RXTE algorithm were the replacement of ST processing with TAM processing, the addition of a second DSS, and the coding of new interfaces to fit the algorithm into the existing, tested flight code. Since the core part of the
Kalman filter code was already tested and working in flight on RXTE, there was a high degree of confidence in it which allowed a 'black box' testing approach to be used for the new algorithm.

\section{Algorithm Development}

The main portion of the algorithm is a discrete. extended Kalman filter. It uses the formulation for covariance propagation, Kalman gain calculation, state estimate update and covariance update given in Gelb.?

\section{System Model}

The system model is described in detail in Andrews and Morgenstern.' In brief, the TRMM Kalman filter has a six component state vector: $\underline{x}=\left[\begin{array}{ll}\underline{\delta \theta} & \underline{\Delta b}\end{array}\right]^{\mathrm{T}}$, where $\underline{\delta \theta}$ denotes the three attitude error angles and $\underline{\Delta b}$ are the three gyro bias errors. The system equation, in state space form, is $\underline{\dot{x}}=\mathbf{F} \underline{x}+\underline{w}$ where

$$
\mathbf{F}=\left[\begin{array}{cc}
-[\underline{\hat{\omega}} \times] & -\mathbf{I} \\
0 & 0
\end{array}\right]
$$

The $3 \times 3$ matrix $[\underline{\hat{\omega}} \times]$ is referred to as a cross product matrix since, for $3 \times 1$ vectors $\underline{a}$ and $\underline{b}, \underline{a} \times \underline{b}=[\underline{a} \times] \underline{b}$, and $\hat{\hat{\omega}}$ is the spacecraft's estimated body rates as measured by the gyros.

The discrete solution to this system of equations is described in Gelb. For TRMM, the form of the state transition matrix is described in Andrews and Morgenstern.'

The state noise vector, $\underline{w}$, is a zero mean, normally distributed random vector, where the state noise covariance matrix is:

$$
\mathbf{Q}=\left[\begin{array}{cc}
T \sigma_{v}^{2} \mathbf{I}+\frac{\mathrm{T}^{3}}{3} \sigma_{u}^{2} \mathbf{I} & -\frac{\mathrm{T}^{2}}{2} \sigma_{u}^{2} \mathbf{I} \\
-\frac{\mathrm{T}^{2}}{2} \sigma_{\mathrm{u}}^{2} \mathbf{I} & \mathrm{T} \sigma_{\mathrm{u}}^{2} \mathbf{I}
\end{array}\right]
$$

where $\sigma_{u}$ is the standard deviation of gyro rate random walk and $\sigma_{v}$ is the standard deviations of the gyro rate white noise, as used in Farrenkopf's gyro noise model." The numerical values of these variables are set based on IRU test data.

\section{Measurement Model}

This section is taken almost entirely from Andrews and Morgenstern', and is repeated here because of the importance of the measurement matrix model effects on the pointing error. Once the system equations are solved, the measurement matrix, $\mathbf{H}$, and the measurement noise covariance matrix, $\mathbf{R}$, must be found to complete the Kalman filter equations. The 
matrix $\mathbf{R}$ is empirically calculated from the noise specifications of the sensors, but the derivation of $\mathbf{H}$ is based on the geometry of the system. The measured vector, provided by either the TAM or the DSS, is $\underline{\tilde{\mathbf{s}}}$. The expected vector, provided by the onboard ephemeris models, is $\underline{\underline{\hat{s}}}$. The residual as represented in the sensor coordinate frame, $Z$, is the difference between these two vectors. The residual can be linearized as a simple subtraction by assuming only a small angle separates $\underline{\tilde{s}}$ and $\hat{\underline{s}}$ :

$$
\begin{aligned}
\underline{Z} & \equiv \overline{\tilde{s}}-\underline{\hat{s}} \\
& =\bar{A}_{\mathrm{bs}} \bar{A}_{\mathrm{lb}} \underline{s}_{1}+\underline{N}-\mathbf{A}_{\mathrm{bs}} \mathbf{A}_{\mathrm{lb}}-\underline{s},
\end{aligned}
$$

The body to sensor transformation matrix, $\mathbf{A}_{\mathrm{bs}}$, is calculated from alignment data, and $\underline{N}$ represents the sensor noise. Since $\mathbf{A}_{\mathrm{lb}}$ is the inertial to true body attitude matrix, $A_{\mathrm{Ib}} \underline{s}_{1}=\underline{s}_{b}$, where $\underline{s}_{b}$ is the vector measured by the sensor, represented in body coordinates. The inertial to estimated body attitude matrix, $\mathbf{A}_{1 \hat{b}}$, can be represented as follows:

$$
\mathbf{A}_{\hat{b}}=\mathbf{A}_{b \hat{b}} \mathbf{A}_{l h}
$$

Here, $\mathbf{A}_{b \dot{b}}$ is the Euler angle matrix corresponding to the attitude errors, $\underline{\delta \theta}=\left[\begin{array}{lll}\delta \theta_{x} & \delta \theta_{y} & \delta \theta_{z}\end{array}\right]^{\mathrm{T}}$, which describe the difference between the true and the estimated body orientations. By assuming small angles, $\boldsymbol{A}_{\mathrm{b} \hat{\mathbf{b}}}$ reduces to

$$
A_{b \dot{b}}=\left[\begin{array}{ccc}
1 & -\delta \theta_{z} & \delta \theta_{y} \\
\delta \theta_{z} & 1 & -\delta \theta_{x} \\
-\delta \theta_{y} & \delta \theta_{x} & 1
\end{array}\right]
$$

When Equations (5) and (6) are substituted into Equation (4), and the attitude error cross product matrix is separated from $A_{b \hat{b}}$, the residual can be written as

$$
\underline{z}=-\mathbf{A}_{\mathrm{bs}}[\underline{\delta \theta} \times] \underline{s}_{\mathrm{b}}+\underline{\mathrm{N}}
$$

Here, the rows of the body to sensor transformation matrix are the sensor basis vectors in the body frame:

$$
A_{\mathrm{hs}}=\left[\begin{array}{l}
\overline{\mathrm{u}}_{\mathrm{sx}} \\
\overline{\mathrm{u}}_{\mathrm{sy}} \\
\overline{\mathrm{u}}_{\mathrm{sz}}
\end{array}\right]
$$

where $\overline{\mathrm{u}}_{\mathrm{si}}$ is the $\mathrm{i}^{\text {th }}$ sensor coordinate frame axis defined in body coordinates. Misalignments, scale factors, or biases introduced to this matrix can cause errors when computing the body to sensor alignment matrix, $\mathbf{A}_{s h}$, if orthogonality is assumed, as shown later. When equation (8) is substituted into equation (7), the full residual is of the form:

$\underline{z}=\left[\begin{array}{cc}\bar{u}_{s x} \times s_{h} & \\ \bar{u}_{s y} \times \underline{s}_{b} & 0_{3 \times 3} \\ \bar{u}_{s z} \times s_{b} & \\ 0_{3 \times 3} & 0_{3 \times 3}\end{array}\right]\left[\frac{\delta \theta}{\Delta b}\right]+\left[\begin{array}{c}\underline{N} \\ \underline{0}-3 \times 1\end{array}\right] \Leftrightarrow \underline{z}=\mathbf{H} \underline{x}+\underline{v}$

This linear form of the measurement matrix, $\mathbf{H}$, must be recalculated each time a new measurement, $s$, becomes available. The measurement noise, $\underline{v}$, is a normally distributed random vector with zero mean and covariance $\mathbf{R}$.

\section{Algorithm Implementation}

The algorithm as implemented in the TRMM flight software does not use the vector/matrix form of these equations. Scalar processing of each measurement component is used to reduce the computational burden. ${ }^{5}$ The TRMM implementation also includes checks on the data in the filter. The first check is made on the availability and quality of the sensor data. For example, if the sun is in the DSS field of view but the measurement is not valid, the filter will not use that DSS measurement to update the state vector. In addition, there is a residual tolerance test that rejects any measurements that yield residuals larger than a set tolerance. These chccks prevent the estimation from using bad sensor data, but, since this does not constitute an algorithm failure, no corrective action is taken.

Originally, there were concerns about processor load when running the Kalman filter, so some assumptions and simplifications were made to reduce the demand. Since the sensor alignment matrix $\mathbf{A}_{b s}$ was assumed to be orthogonal, the columns of $\mathbf{A}_{b x}$ were used as the rows of $\mathbf{A}_{s h}$. The proper way to compute the rows of $\mathbf{A}_{s h}$ is to take the rows of the inverse of $\mathbf{A}_{b s}$. This transpose shortcut affected the calculation of the measurement residual and the calculation of the measurement matrix $\mathbf{H}$. The effects of this shortcut will be discussed later.

There are also three Failure Detection and Correction (FDC) checks designed specifically to monitor the Kalman filter algorithm. Two checks monitor the covariance matrix for divergence and positive semidefiniteness. The third test ensures that the actual residual remains within $3 \sigma$ of the expected value of the residual. For all three tests, the ACS software autonomously performs the same actions. First, the software stops updating the attitude quaternion and the 
gyro drift with the flawed estimate; then, it commands the spacecraft to a power and thermal safe attitude.

\section{Flight Results}

Initialization and Earth Acquisition

The Kalman filter was first enabled when TRMM was still in Sun Acquisition Mode holding the spacecraft inertially fixed in a power and thermal safe attitude with the spacecraft $+\mathrm{X}$-axis $16.5^{\circ}$ from the sunline. In this mode, the spacecraft is controlled directly off the CSSs and the IRUs; the Kalman filter output is not used in the control loop, which provides an ideal initialization configuration.

The DSS residuals for the first 32 hours are shown in Figure 1. The flat line portions in this figure are periods when the sun is not in the respective DSS field of view (FOV). The multi-axis slew to acquire the nadir-pointing attitude is clearly seen, as is the first sunlight in DSS2. The DSS residuals are approximately zero mean, but they are not the white noise processes modeled by the filter equations. The magnitude of this modeling error has implications for setting the proper sensor noise parameters in the filter and for filter estimation accuracy.

In addition, the spikes in the residuals at the edge of the field of views are caused primarily by stray light or glint affecting some measurements. These spikes can get into the measurement updates and cause the gyro bias estimate to oscillate at approximately orbit period. A solution to this problem is discussed later.

The TAM residuals are shown in Figure 2. The residuals are approximately zero mean, but they aren't the white noise processes modeled in the Kalman filter. Earth Acquisition doesn't change the residual pattern noticeably. Once the sun measurement from both sun sensors is used, there is a noticeable increase in the oscillation of the TAM residual. This indicates a misalignment between the two DSS heads, a misalignment between the TAM and DSS2, or a modeling error in the TAM that shows up when the spacecraft is rotating at 1 rev/orbit. Before hour 28 , the TRMM Microwave Imager (TMI) instrument was turned on. It rotates at $31 \mathrm{rev} / \mathrm{min}$, and generates a rotating magnetic field that is not modeled in the filter. This looks like an increase in the noise level of the residual during the first four hours of the plot.

This residual signal is not zero mean, and is not white noise. Some of the errors are due to an old calibration matrix and an old magnetic field model onboard. The steps taken to describe and to reduce these errors will be discussed later in this paper.
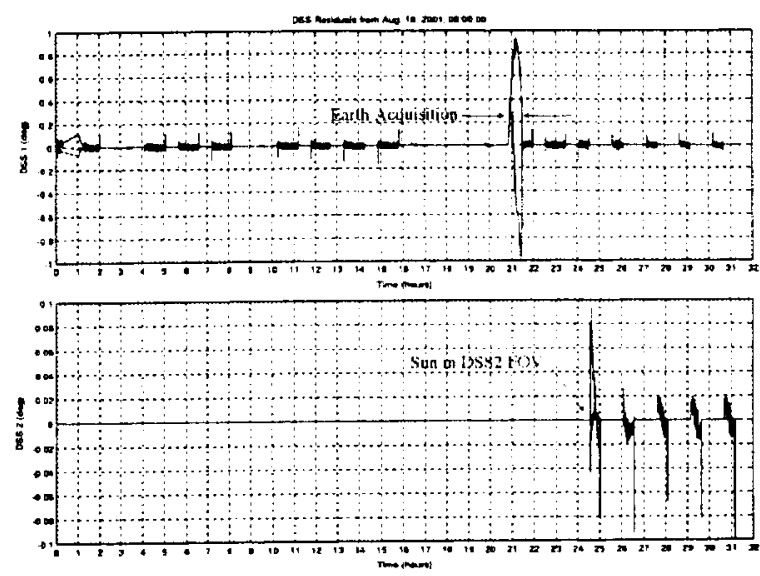

Figure 1: Initialization and Earth Acq: DSS Residuals

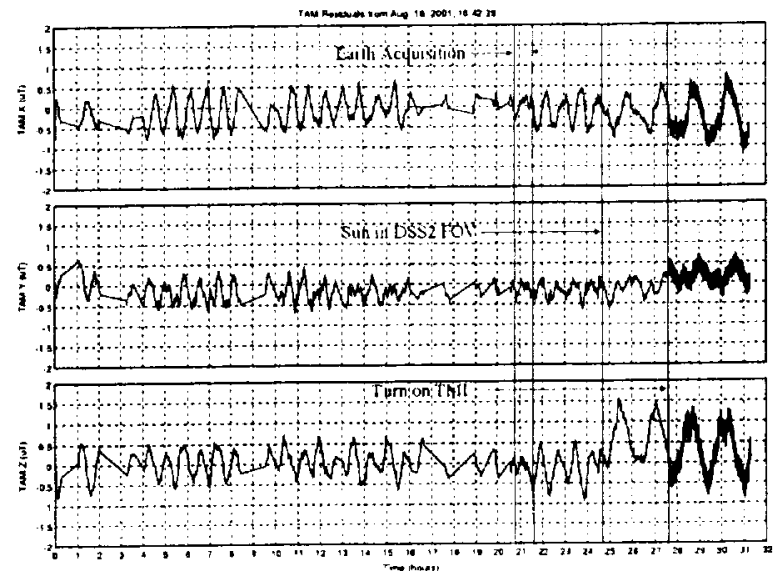

Figure 2: Initialization and Earth Acq: TAM Residuals

\section{Mission Mode}

Since the Kalman filter was enabled, it has performed the required Earth Acquisition, Yaw Acquisition, and Mission Mode attitude determination. It has controlled the spacecraft to a nadir-pointing attitude, and has worked during Delta $\mathrm{V}$ maneuvers and $180^{\circ}$ Yaw Maneuvers. After TRMM entered mission mode. several efforts were made to independently verify the attitude performance. First, close examination of the horizon sensor data was pursued; however, the behavior of that sensor at the new altitude was not well understood, and could not be used to calculate any attitudes. Second, independent ground calculation of the attitude was accomplished for selected orbits using the same sensor compliment as used in the flight Kalman filter. This gave different results by a few tenths of a degree for some orbits, however the estimated accuracy was initially not much better than the error magnitude. Third, calculation of the roll attitude from the Precipitation Radar (PR) science data products was pursued." The PR is used measure the vertical structure of rainfall in 250 meter bins, but it also detects the surface echo. Roll attitude could be estimated from the 
diflerence in the measured surface distances between the right and left sides of the symmetric cross track scan.

\section{Attitude Analysis}

A sample of the roll history estimated from the PR data for one orbit is shown in Figure 3(a). There are data gaps over land since it was decided to avoid topographical effects and only compare the radar surface echo distances over sea surfaces. The computed roll is effectively with respect to a geodetic frame (vertical perpendicular to the Earth geoid), and a bias term was taken out accounting for the PR alignment. Also shown in Figure 3(b) is the roll attitude reported onboard, which is also with respect to a geodetic frame (referred to as the tip frame, computed onboard for consistency with a horizon bisector model for the original control mode). Since the spacecraft controll is based to the onboard estimate, the reported roll is generally very close to zero. Certain very minor disturbances show up regularly, with the ones in this example being due to, in time order, effects of solar array tracking initializing before shadow exit, high gain antenna initializing tracking and stowing, and solar array feathering after shadow entry. Note that the difference in the scales of the two plots is more than two orders of magnitude.
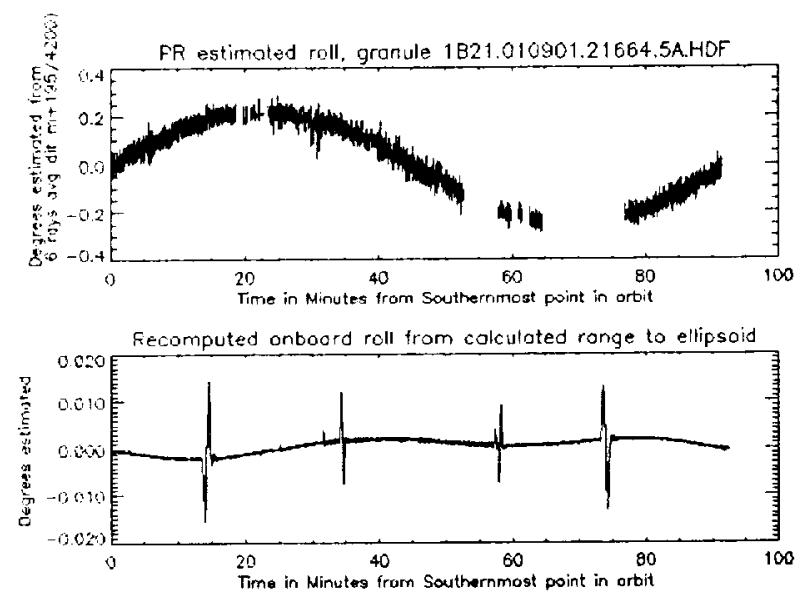

Figure 3: Roll Attitude Comparison

An orbit period sine wave for errors in onboard roll turned out to be a consistent pattern. The amplitude and phase of the sine wave pattern varied little over each day, but gradually changed over days and weeks (with one exception--the phasing shifted by $180^{\circ}$ as a result of $180^{\circ}$ yaw maneuvers every two to four weeks).

It was soon understood that gyro propagation of any error in the initial roll attitude could be expected to follow this sine-wave-like pattern, as the same error pattern was demonstrated in sample ground solutions. This is because the one revolution per orbit rotation of the spacecraft for Earth pointing is well tracked by the gyros, but the mean rotation axis is offset with various initial roll or yaw values. Roll and yaw are coupled similar to the coupling for spin stabilized spacecraft with a near-orbit-normal rotation axis. Thus the PR data was fit with an orbit period sine wave and seemed to give a reliable estimate of the average coupled roll/yaw error per orbit, to an accuracy of hundredths of a degree. Trends in this error were tracked

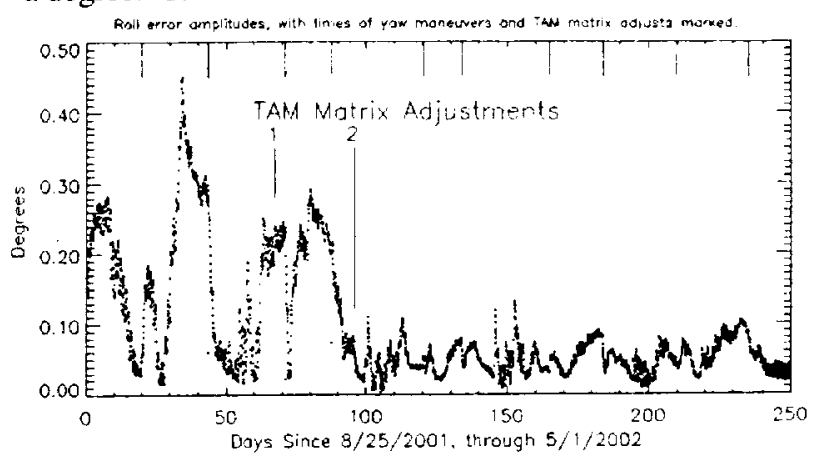

Figure 4: Roll Attitude from PR Data

Meanwhile it was also understood that onboard ephemeris errors were affecting the pointing, since the spacecraft depends on the onboard orbit propagation to compute the Earth pointing reference frame. The previous control method depended directly on the ESA data for Earth pointing, and the onboard ephemeris could be less accurate for tasks like High Gain Antenna pointing. On September 27 th, an onboard vector update failed the 50 kilometer along-track consistency check, and errors were as high as 80 kilometers before a new vector was loaded. This corresponded to about $0.7^{\circ}$ pitch error in the reference frame. Soon after this event, it was decided to load vectors twice per day instead of once per day, and efforts were made to better track the ephemeris errors and improve the onboard propagation. Ephemeris error effects and improvements will be discussed further later.

The rollyaw errors reached a peak of nearly $0.5^{\circ}$ around the same time as the large onboard ephemeris errors, so by October 2001 it was clear there were some significant errors in the onboard attitude estimate, relative to the initial mission goal of $0.2^{\circ}$. The magnetometer data was the most suspect for various reasons: it had the largest measurement residuals, and analysis of the phasing the roll errors from the PR science data indicated that the error in the average body rotation axis was mostly in a direction around the sunline, which the DSS could not measure.

\section{Attitude Error Fixes}

By the end of October, the TAM had been recalibrated to update the alignments, scale factors, and biases, and the contamination matrix (used to remove the MTB output from the TAM measurement) had been recalculated This improved the TAM residual characteristics, but not enough to improve the attitude performance as measured 
by the PR (label 1 in Figure 4 ). Previously, the DSS residual tolerance had been reduced from $1.5^{\circ}$ to $0.5^{\circ}$ soon after the filter initialization to remove oscillations in the gyro bias estimate and jumps in the attitude estimates. The tolerance was further reduced to $0.2^{\circ}$ on November 13, but that still wasn't small enough to reduce the effects of the relatively large DSS data spikes near the edges of the DSS fields of view.

An ACS on-board software error in TAM reference trame conversions, which affects the Kalman Filter TAM residuals, was discovered on November $16 .{ }^{10}$ The ACS flight software computes the transformation from sensor frame to body frame for the TAMs ( $\mathbf{A}_{b s}$, a $3 \times 3$ matrix for each TAM) assuming orthogonality, as discussed previously. However, it turns out this assumption is not correct for TRMM because the Flight Dynamics Facility (FDF) calibrations incorporate scale lactors and misalignments." The result is that the residual calculation can be off by as much as $0.4 \mu \mathrm{T}$ $\left(\sim 0.5^{\circ}\right)$ and the $\mathbf{H}$ matrix alignment could be off by as much as $0.5^{\circ}$. Thus, the TAM attitude updates are the wrong size in the wrong direction, and this resulted in attitude estimation errors around the sunline. The DSS measurements were accurate to a few hundredths of a degree perpendicular to the sunline, based on the size of the DSS residuals.

The decision was made to modify ACS Flight Software Tahle 58 (TAM-to-Body Alignment Matrix) by uplinking the transpose of the inverse of the current version rather than by creating an ACS flight software patch. An updated version of ACS System Table 58 was uplinked on November 28, 2001, in an effort to improve the Kalman filter pointing. The only other portions of code that use this table are momentum unloading and a deterministic attitude determination algorithm. Simulations showed that this change is small enough that it does not cause any noticeable effects with those tasks." Figure 4 clearly shows the improvement in roll pointing after correcting the onboard alignment matrix (label 2 in Figure 4).

The effect on the TAM residuals was immediate and dramatic. The plot of TAM residuals in Figure 5 is centered on the alignment update time.

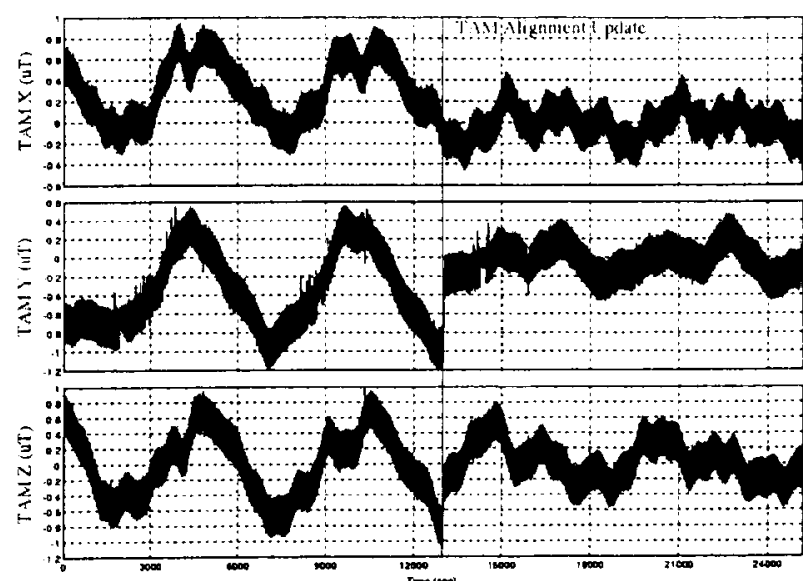

Figure 5: TAM Alignment Update: TAM Residuals

Table 1 shows how much improvement there was in each axis, based on the data shown in Figure 5. Most significantly, the mean TAM residuals are now one-fifth to one-thirtieth their pre-update values. There is still some long-term, somewhat periodic characteristic in the TAM residual data. That is probably due to the onboard software using the 1995 International Geomagnetic Reference Field (IGRF) model. There is a software patch in testing that will update the magnetic field model to a 2000 IGRF. This can reduce attitude estimation errors by as much as $0.6^{\circ}$ over 25 years. ${ }^{12}$

Table 1: TAM Residual Statistics

\begin{tabular}{|c|c|c|}
\hline & mean $(\mu \mathrm{T})$ & $\begin{array}{c}\text { standard deviation } \\
(\mu \mathrm{T})\end{array}$ \\
\hline Before & 0.35 & 0.30 \\
TAM X & -0.34 & 0.42 \\
TAM Y & -0.05 & 0.45 \\
TAM Z & & 0.18 \\
\hline After & 0.02 & 0.20 \\
TAM X & -0.01 & 0.28 \\
TAM Y & -0.01 & \\
\hline
\end{tabular}

The attitude corrections computed by the Kalman filter are shown in Figure 6. After the alignment update, the attitude corrections during eclipse (for example, the short space centered at 15000 seconds in the plot) are now much smaller than the corrections during the same period before the update, especially in the $\mathrm{Y}$ axis. The attitude error introduced by biased TAM measurements has now been reduced. 


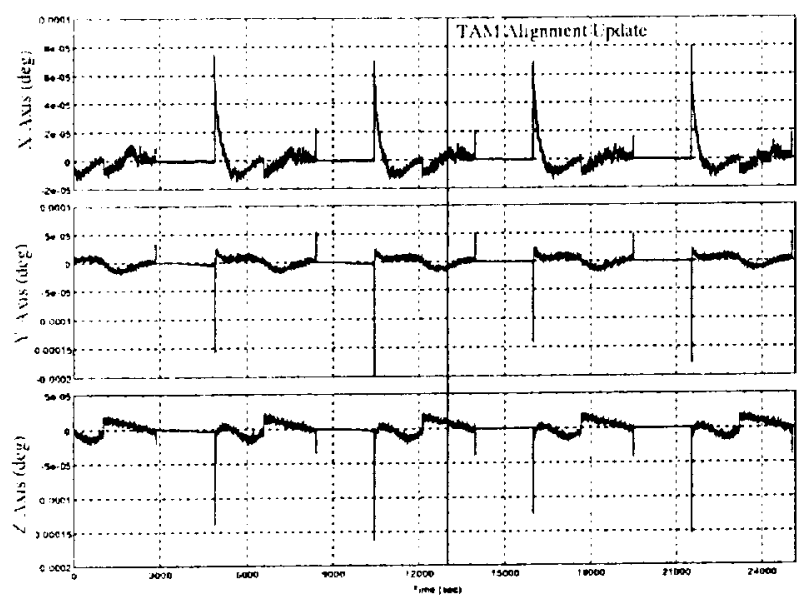

Figure 6: TAM Alignment Update: Attitude Corrections

As expected, the DSS residuals showed little change (see Figure 7). The Kalman filter has already taken out the DSS measurement errors, and because the DSS is more accurate and the DSS measurement is weighted more than the TAM measurement, the TAM improvements have no effect on the DSS residuals.

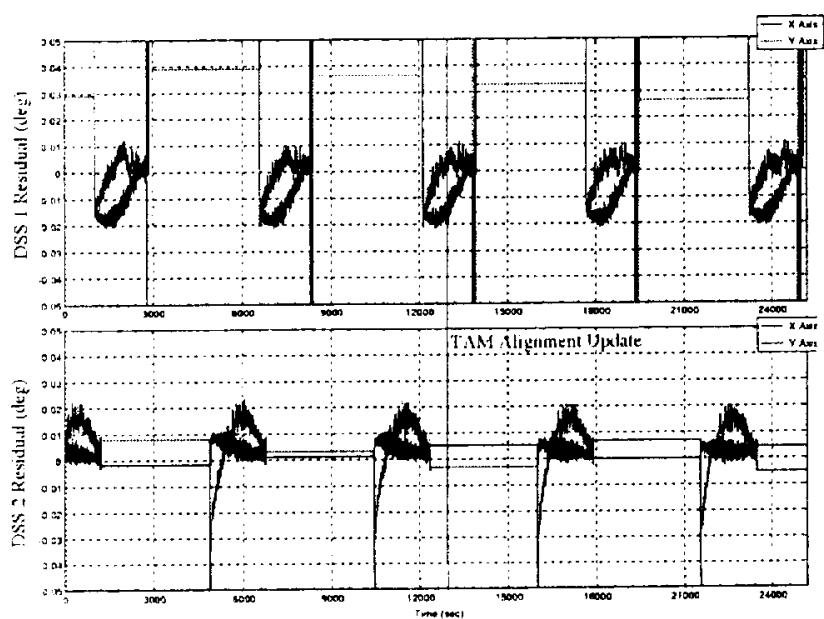

Figure 7: TAM Alignment Update: DSS Residuals

\section{Ephemeris Error Effects on Attitude}

When TRMM is using the Kalman filter for attitude determination. onboard ephemeris errors show direct effects on pitch and secondary effects on the roll and yalw attitude. Details about the causes of ephemeris errors are beyond the scope of this paper, but the error magnitudes are shown, improvements implemented are noted, and the effects on attitude discussed here.

Briefly enumerated, the following changes to the ephemeris Extended Precision Vector (EPV) uplinks have been implemented since the switch to the Kalman Filter:
1) On September 28, uplinks were switched from once per day at 20:00 UT to twice per day at 08:00 and 20:00.

2) Starting November 9th, a drag correction factor was included in the uplinked parameters,

3) Starting January 7th 2002, EPVs were loaded at times within $\sim 45$ minutes of 08:00 and 20:00, but with the exact time chosen to give an optimal geopotential match with the ground ephemeris model, based on results reported by Beckman ${ }^{13}$

4) After January 29th, improved modeling of the expected burns was included for ephemeris prediction generation on days of burn execution.

5) After confidence was gained in the results from the last two corrections, onboard vector updates were switched back to once per day after March 5, 2002.

The principal ephemeris errors are in the along track direction, and typically the errors will grow until the next EPV uplink. Since the onboard ephemeris is used to generate a target Earth pointing attitude for the spacecraft, along track errors translate directly to pitch errors. This can be seen in immediate pitch changes onboard at the EPV load times. There is a $0.1^{\circ}$ pitch error for every 11.83 kilometers of along track error at mean orbit radius of about 6780 kilometers. A record of the along track errors is shown in Figure 8, along with the labels for the five activities listed above.

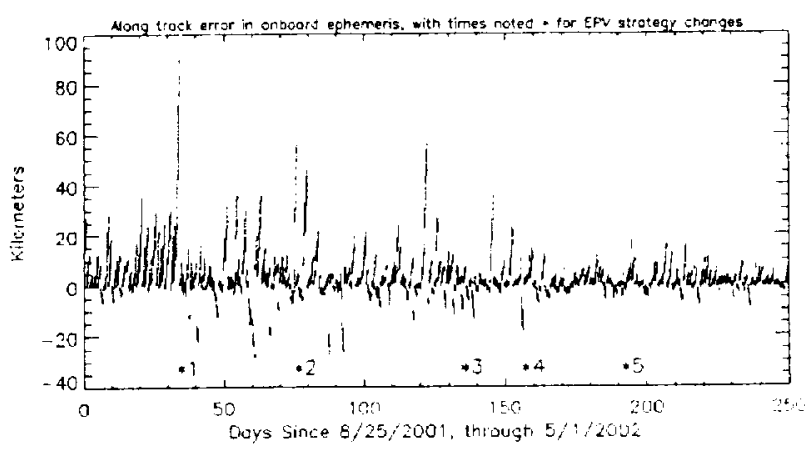

Figure 8: Along-track errors in onboard ephemeris

Note the onboard error growth has two components; one is due to onboard propagation error, and another is due to the ground ephemeris prediction errors for the EPV uplink time. The ground computed ephemeris is computed once each day around 15:00 UT along with the definitive ephemeris for the previous day. Thus the 20:00 load is based on that day's delivery, and at 08:00 the next day it is still based on the previous day's delivery. Inasmuch as the ground-computed prediction is in error, the onboard orbit is in error even right after the load. The most significant initial position errors happened with the 08:00 loads due to the EPV prediction being further from the last definitve orbit solution, and these can be seen in Figure 8 as the line segments that start away from zero. Most often, these large errors occurred on days after orbit adjust burns 
The onboard propagator thereafter affects the growth rate in the along track errors, depending on the vector uplinked.

An association became apparent whereby the larger excursions in ephemeris errors often correlated with relatively rapid shifts in the roll/yaw pointing errors. Further, the correlation was particularly distinct at higher magnitude solar beta angles (i.e. at high Sun elevations from the orbit plane). The effect can be seen by comparing Figures 4 and 8 . Maximum values of beta occur about midway between the yaw maneuver times that are marked at the top of Figure 4, and some large ephemeris errors are aligned with spikes in the roll amplitude at these times. Also, for example, the period of consistent daily positive error along track before September 28 th $\left({ }^{*} 1\right.$ in Figure 8$)$ undoubtedly helped push the amplitude of the roll/yaw error higher.

This association is illustrated more clearly if we consider both the phase as well as the amplitude information from the sine wave signal in the roll errors and associate directions to these changes. To do this we computed the direction of the Sun in the orbit plane, where local noon occurs with respect to the beginning of each full orbit data granule. We then calculated the phase angle around the orbit, with respect to the local orbit noon, where the maximum roll angle of the sine wave occurs. This phase tended to be either $90^{\circ}$ or $270^{\circ}$, with the phase switching rapidly past $180^{\circ}$ as the roll amplitude got very low. This has a direct interpretation considering TRMM primarily rotates about the pitch axis at 1 revolution per orbit: the angular offset of the TRMM rotation axis from orbit normal is given by the amplitude of the sine wave, and the direction of the offset is given by the phase. Thus the $90^{\circ}$ or $270^{\circ}$ phase angles with respect to the Sun indicate that our estimate of the TRMM rotation axis from PR data tends to drift mostly about the Sun direction.

This drift is illustrated in Figure 9, along with along track orbit errors for a period in January 2002. The beta angle is zero (the Sun is in the orbit plane) at the times of the two yaw maneuvers marked, and the Sun reached ibout 55 degrees below the orbit plane in the time span in between. There were three notable spikes in along track error during the period of high beta -- two up and one down, and the spin axis drifted the opposite direction about the Sun in each case -- twice down and once up. Conversely, ephemeris errors on days with near zero beta angle had little effect on attitude.

There is some evidence of a diurnal cycle in the drift at higher beta magnitudes, and apparently the sensitivity (1) the orbit errors is greater at certain times of day. We have not modelled these effects, but see reasons to expect sensitivity. Errors in the along track orbit have

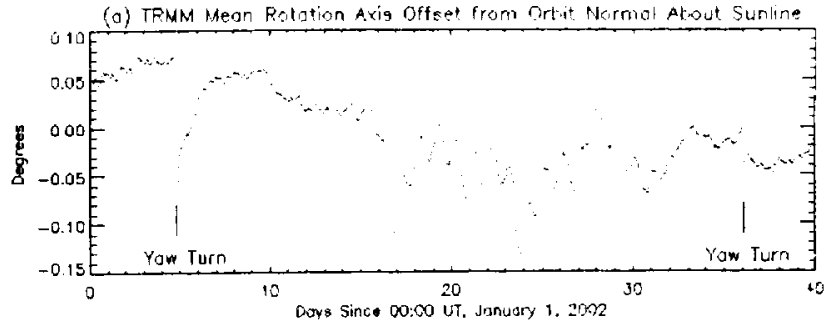

(b) TRMM Onboord Ephemerio Along Track Error

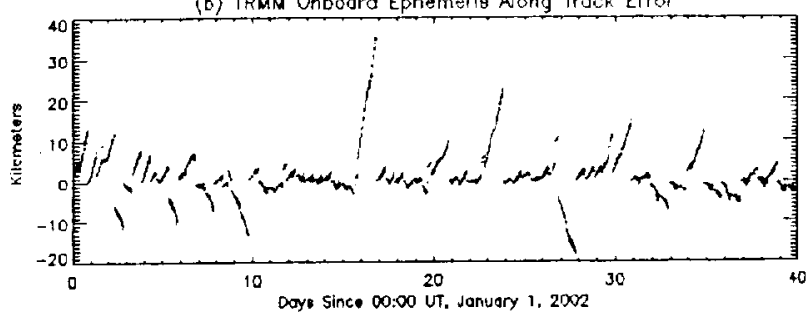

Figure 9: Rotation Axis Offset and Along Track Error

two effects on computed magnetometer measurement residuals: the magnetic field model is calculated for the wrong orbit position and for the wrong body orientation (there is a pitch targetting frame error, as previously discussed). Errors in the calculated magnetic field will tend to cause incorrect updates to the mean rotation axis about the sunline. The DSS data maintains the mean rotation axis estimate at the proper separation from the sunline. At high beta magnitude, rotations about the sunline couple pitch with roll and/or yaw depending on the position in the orbit.

\section{Lessons Learned}

The improvements in the TRMM Kalman filter fall into three basic categories: operational issues. performance issues, and implementation features. Operational issues include transitions to the backup mode, testing, software design, and data flow. Performance issues pertain to properly tuning a Kalman filter so that it functions effectively with real sensors. Implementation features concerns algorithm and software design.

\section{Operations issues}

Since the TRMM Kalman filter was added late in the testing cycle, the concern about onboard processing power forced the software design to an either/or mindset. Either the ESA processing or the Kalman filter could be run, but not both. Assuming the filter only had to replace the ESA functions meant that the filter's performance during other cases, such as thruster maneuvers, was not thoroughly considered. This led to several logistical oversights in the filter design. The software propagates the attitude estimate during thruster maneuvers, but it does not propagate the filter covariance. Thus, after completing the thruster burn, the covariance gives an incorrect indication of the accuracy of the attitude estimate. TRMM has extremely 
stable IRU drift rates however, and the size of the error at the end of a burn has not warranted a reinitialization of the Kalman filter. Yaw maneuvers, thruster burns, and flight software table changes have occurred without the need for the Kalman filter to be reinitialized because of the stability of the IRUs.

In the absence of an ESA for Earth pointing control, ephemeris prediction for onboard use becomes much more important and represents a challenge. This is especially true for a satellite executing frequent orbit adjustments. It's also more of a challenge during a period of high solar activity for a low Earth orbiting satellite since the drag can change fairly quickly. The main component of concern is the along-track prediction which is the hardest to accurately forecast, and which directly affects pitch pointing. However, users of sun sensor/magnetometer combinations in Kalman filters should be aware that ephemeris errors could have effects on roll and yaw as well. Accurate burn modeling and good, ground computed predictions are most important for attitude determination accuracy, but reliable onboard propagation after the vectors are loaded is important too. On TRMM, some ephemeris concerns might have been overlooked initially because the Kalman filter was only developed for backup use and wasn't originally intended for maintaining the original pointing requirements. Onboard ephemeris accuracy was less important under the original control law.

It is very useful to have alternate sources for checking the onboard attitude performance. The TRMM ESA data could not be used at the higher altitude, so the science radar data application became very useful in showing the roll performance. The use of the same attitude sensors in ground processing software for recomputing the attitude was also helpful, and eventually provided key insights from differences in the results and magnetometer residual characteristics.

The processing from the science data system was set up to provide trending using all the selected daily data which helped give a global picture of the performance, and catch any unusual transient events. Other ground computations were set up to use selected shorter data spans, and while representative spans are undoubtedly usetul and applicable to solving problems, additional information is often illustrated in long term trends. For example, various aspects of the solar beta angle sensitivity need a long-term view to be seen. Also, the long-term trends could point to specific spans of interest that might be analyzed further by other tools. For example, the periods of larger ephemeris errors and changes in attitude were examined more closely after being highlighted in the long-term trends. Large archive datasets and product generation systems like the TRMM Science and Data Information System (TSDIS) are common for many Earth science missions today, and the data can be used for additional flight dynamics analysis as well as for science products as a need or interest arises. Regular data reprocessing with improved science algorithms is a planned part of the TSDIS effort, and further analysis and improvement of the attitude estimation (affecting image pixel geolocation) is now under review for future reprocessing.

\section{Performance issues}

The Kalman filter models assume zero-mean white noise measurement residuals, which is not true of either the DSS residuals or the TAM residuals. The largest sources of error from the DSS measurements are the spikes at the edges of the field of views. These will be mostly removed when the DSS residual tolerance is reduced to its final value of $0.05^{\circ}$. The largest source of error from the TAM measurements is the modeling error that result from using an old magnetic field model onboard. As mentioned previously, the onboard magnetic field model should be updated to the latest epoch to remove any remaining modeling errors. In addition, the new ephemeris loads, with more accurate initial conditions, will help the post-burn attitude and also the pitch command error at upload time.

The Kalman filter parameters could be retuned to give better performance, or less sensitivity, to DSS measurements, for example, but that is a second-order change. The primary concern should be eliminating the gross errors like misalignments and modeling errors. There are currently no plans to retune the TRMM Kalman filter; the state noise model parameters were taken from gyro test data, and the measurement noise parameters are based on the current sensor performances. Unless the sensor residuals change drastically, the current filter settings will not change.

\section{Implementation features}

The flight software developers should avoid hardcoding values; a table-based design allows parameters to be changed with a simple uplink. Software patches require significant development and testing, and risk the safety of the spacecraft. For example, some of the DSS misalignment error was calibrated out of the data by modifying table values, but updating the magnetic field model to a new epoch will require a software change.

Also, there should be a way to limit, onboard, the DSS FOV used in the Kalman filter. Currently, this is done by limiting the size of the DSS residual tolerance, but this somewhat arbitrarily limits the area of the DSS that is used. It would be better to more directly limit the area of the DSS that is acceptable to use.

A more subtle improvement is probably TRMMspecific. The FDF team calibrates and aligns the TAM by including the misalignments and scale factor errors in the $3 \times 3$ alignment matrix. This removes the 
orthogonality of that matrix, and makes the "transpose = inverse" assumption false. There are two ways to correct this. First, the calibration could break out the different parameters into separate data files to be loaded to separate tables on the spacecraft. The second solution is to explicitly calculate the inverse of the transformation matrix and use its rows, instead of assuming that the rows of the transpose of the original matrix are the same as the rows of the inverse. TRMM worked around this problem by loading the transpose of the inverse to the original matrix, so that the columns of that matrix are the rows of the inverse of the original. The designers and users of the ACS should each understand what the other assumes for data formats and contents, and how it will be used.

\section{Conclusion}

A backup attitude determination method has been devcloped for and enabled on the Tropical Rainfall Measuring Mission spacecraft. This algorithm uses data from a three-axis magnetometer, two digital sun sensors, and an accurate gyro package in an extended Kalman filter, and employs onboard ephemeris and geomagnetic field models. Flight results using the Precipitation Radar instrument data demonstrate Earth pointing accuracies to less than $0.2^{\circ}(3 \sigma)$ in the roll axis after proper sensor calibration and ephemeris processing updates, without direct sensing of the Earth. This meets the pointing requirement for the ESA-based controller.

The algorithm has provided attitude determination accurate enough to enable TRMM to continue its primary mission of measuring tropical rainfall. All the control modes have been exercised, and the Kalman filter works properly in all cases. There is some work remaining to further reduce the remaining errors in the attitude determination and control, but at this point, the spacecraft is operating safely and is meeting requirements.

\section{Acknowledgements}

The authors would like to thank Gregory Natanson and Joe Hashmall for providing ground attitude determination support, magnetometer calibration, and for investigating all possible error sources in the Kalman filter. In addition, we would like to thank Steve Slojkowski for his support in improving the ephemeris processing to give more accurate EPVs, and for other attitude and trending support. Landis Markley also spent much time helping us analyze the problem and searching for a solution. The TRMM Flight Operations Team was invaluable in getting us data, trending data, and in modifying existing procedures to improve the performance of the TRMM spacecraft and ground system. Also, we'd like to thank John Kwiatkowski for implementing the PR trending of the roll errors.

\section{References}

${ }^{1}$ Andrews, S. F., Morgenstern, W.M., "Design, Implementation, Testing, and Flight Results of the TRMM Kalman Filter", AIAA Paper 98-4509. Aug. 1998.

${ }^{2}$ TRMM FOT, TRMM Weekly Report, Aug. 13, 2001 - August 19, 2001, available online at http://trmm-

fot.gsfc.nasa.gov/WEEKLY/files/Weekly\%20(01\%2022 5-231).pdf

${ }^{3}$ J.L. Crassidis, S.F. Andrews, F.L. Markley, K. Ha, "Contingency Designs for Attitude Determination of TRMM", Flight Mechanics/Estimation Theory Symposium, 1995.

${ }^{4}$ Hashmall, J.A., Rokni, M., Sedlak, J., Harman, R., Ketchum, E., How Accurate an Attitude Can We Get Using Magnetometers?, Flight

Mechanics/Estimation Theory Symposium, 1994.

5 Murrell, J.W., "Precision Attitude Determination for Multimission Spacecraft," AIAA Paper 78-1248, Aug. 1978.

${ }^{6}$ Lefferts, E.J., Markley, F.L., and Shuster, M.D., "Kalman Filtering for Spacecraft Attitude Estimation," Journal of Guidance, Navigation, and Control. Sept.Oct. 1982.

${ }^{7}$ Gelb, A. (ed.), Applied Optimal Estimation, The MIT Press, Cambridge, MA, 1974.

${ }^{8}$ Farrenkopf, R.L., “Analytical Steady-State Accuracy Solution for Two Common Spacecraft Attitude Estimators," Journal of Guidance and Control, Vol. 1, No. 4, July-Aug, 1978. pp.282-284.

${ }^{9}$ J. Kwiatkowski, S. Bilanow, J. Stout, "Estimating TRMM Spacecraft Attitude Errors Using the Precipitation Radar," accepted in Proceedings of IEEE 2002 International Geoscience and Remote Sensing Symposium. Toronto, Canada. June 2002.

10 TRMM FOT, TRMM Weekly Report, Nov. 12 , 2001 - Nov. 18, 2001, available online at http://trmmfot.gsfc.nasa.gov/WEEKLY/files/Weekly\%20(01\%2031 6-322).doc.

11 TRMM FOT, TRMM Weekly Report, Nov. 12 , 2001 - Nov. 18, 2001, available online at http://trmmfot.gsfc.nasa.gov/WEEKLY/files/Weekly\%20(01\%2033 0-336).doc.

${ }^{12}$ Hashmall, J.A., Sedlak, J., "The Use of Magnetometers for Accurate Attitude Determination," Space Flight Dynamics Symposium. Darmstadt. Germany, June, 1997.

13 M. Beckman, "Reducing On-Board Computer Propagation Errors Due to Omitted Geopotential Terms By Judicious Selection of Uploaded State Vector", Flight Mechanics/Estimation Theory Symposium, 1996.

10

American Institute of Aeronautics and Astronautics 